\title{
Caracterização do material particulado suspenso no ar de Cuiabá-MT no período de queimadas
}

\author{
Characterization of suspended particulate \\ matter in the air of Cuiaba-MT-Brazil \\ during the forest burning period
}

\author{
Aline Santiago ${ }^{1}$, Rogério Junqueira Prado ${ }^{2}$, \\ Paulo Modesto Filho ${ }^{1}$, Rafael Viana Alonso ${ }^{3}$
}

\footnotetext{
${ }^{1}$ Universidade Federal de Mato Grosso (UFMT), Programa de Pós-graduação em Engenharia de Edificações e Ambiental (PPGEEA), Av. Fernando Corrêa da Costa, 2367, CEP 78060-900, Cuiabá - MT. e-mail: alinetutusan@ hotmail.com; modesto@ufmt.br

${ }^{2}$ Universidade Federal de Mato Grosso (UFMT), Instituto de Física, Av. Fernando Corrêa da Costa, 2367, CEP 78060900, Cuiabá - MT. e-mail: rjprado@ufmt.br

${ }^{3}$ Universidade Federal de Mato Grosso (UFMT), Departamento de Química (ICET), Av. Fernando Corrêa da Costa, 2367, CEP 78060-900, Cuiabá - MT. e-mail: rafaelalonso_22@ hotmail.com
}

\section{RESUMO}

A caracterização química e morfológica do material particulado suspenso no ar da cidade de Cuiabá - MT, no período de queimadas do ano de 2012, foi realizada utilizando-se as técnicas de Microscopia Eletrônica de Varredura acoplada a um sistema de Microanálise de Raios X (SEM/EDS) e Fluorescência de Raios X por Dispersão em Energia(EDXRF). Deve ser ressaltado que são poucos os estudos realizados sobre o tema, particularmente para a cidade de Cuiabá e região. Com o uso das técnicas de SEM/EDS foi possível classificar o material particulado em quatro diferentes grupos, definidos em função de suas semelhanças morfológicas e composição química: aglomerados, placas, fibrosas e arredondadas. Por EDXRF foram identificados e quantificados 10 elementos químicos ( $\mathrm{Al}, \mathrm{S}, \mathrm{Cl}, \mathrm{K}, \mathrm{Ca}, \mathrm{Fe}, \mathrm{Cu}, \mathrm{Zn}, \mathrm{Sr}$ e $\mathrm{Ba}$ ), predominando aqueles característicos de ressuspensão do solo e queimadas $(\mathrm{Al}, \mathrm{Fe}, \mathrm{Ca}, \mathrm{K}$ e $\mathrm{Cl}$ ), além dos vinculados às emissões veiculares e industriais $(\mathrm{Zn}, \mathrm{S}, \mathrm{Cu})$. As concentrações de material particulado em suspensão obtidas no mês de setembro, após longo período de seca, atingiram $306 \mu \mathrm{g} / \mathrm{m}^{3}$ de ar, superando o limite primário estabelecido pelo Conselho Nacional do Meio Ambiente (CONAMA), evidenciando a má qualidade do ar no período. Além disso, as partículas fibrosas identificadas neste trabalho não haviam sido relatadas anteriormente, sendo aparentemente características da região, e apresentando composição química condizente com forte influência de queimadas em sua formação.

Palavras-chave: material particulado; caracterização química e morfológica; queimadas.

\section{ABSTRACT}

The chemical and morphological characterization of the particulate matter found into the air of Cuiaba Municipality - Mato Grosso State - Brazil, during the forest burning period of 2012, was performed by Energy Dispersive X-Ray Fluorescence (EDXRF) and Scanning Electron Microscopy with X-ray Micro-analysis (SEM/EDS) techniques. It should be noted that there are few studies on the subject, particularly for Cuiaba Municipality and region. Using SEM/EDS techniques was possible the classification of the particulate matter in four different groups, defined by morphological similarity and chemical composition: agglomerates, plates, fibers and spheroids. Ten chemical elements ( $\mathrm{Al}, \mathrm{S}, \mathrm{Cl}, \mathrm{K}, \mathrm{Ca}, \mathrm{Fe}, \mathrm{Cu}, \mathrm{Zn}, \mathrm{Sr}$ e Ba) were identified and quantified by EDXRF, mainly showing the existence of re-suspension of soil particles by mechanical processes and fires ( $\mathrm{Al}, \mathrm{Fe}, \mathrm{Ca}, \mathrm{K} \mathrm{e} \mathrm{Cl})$, and in minor extent vehicle and industrial emissions ( $\mathrm{Zn}, \mathrm{S}, \mathrm{Cu}$ ). Concentrations of particulate matter found in air, after long drought, attained $306 \mu \mathrm{g} / \mathrm{m}^{3}$ of air, exceeding the physical limit established by the Brazilian environmental national council (CONAMA) and showing the poor quality of the local air in the period. Furthermore, the fibrous particles identified in this study have not been previously reported, being apparently characteristic of the region, and presenting chemical composition consistent 
with a strong influence of fire on their production.

Keywords: particulate matter; chemical and morphological characterization; fires.

\section{INTRODUÇÃO}

Mato Grosso tem registrado um grande número de focos de queimada durante o período de seca, fato que coloca o estado em uma posição de evidência no contexto nacional, tendo sido citado nos últimos anos como uma das unidades federativas com maior número de registros de queimadas [1], o que contribui de forma expressiva na emissão de gases e material particulado, alterando profundamente as características químicas e físicas da atmosfera [2, 3$]$, resultando em impactos ambientais e de saúde tanto em escala local quanto global.

Além disso, Mato Grosso ainda sofre grande influência das emissões oriundas da Amazônia, por meio da movimentação da Massa de ar Equatorial Continental (MEC) que se origina na região Amazônica, e acaba por influenciar a atmosfera de toda a porção interiorana da América do Sul, se deslocando por meio de correntes de noroeste, oeste e sudoeste a partir do seu centro de ação [4].

Dentre os poluentes atmosféricos, o material particulado apresenta grande relevância devido à sua complexidade em termos de composição química e propriedades físicas, abrangendo uma grande classe de poluentes constituída de poeira, fumaça e todo material sólido e líquido que, devido ao pequeno tamanho, mantém-se suspenso no ar [5]. Materiais particulados estão associados a um grande número de problemas de saúde quando em alta concentração $[\underline{6}, \underline{7}, \underline{8}]$ motivo pelo qual tem despertado grande interesse científico, tanto por sua relação com efeitos adversos à saúde humana, quanto pelos efeitos causados ao ambiente.

O material particulado emitido pelas queimadas possui menor dimensão e densidade, o que prolonga sua permanência no ar, causando as cortinas de fumaça. Ainda, a queima de vegetação produz temperaturas elevadas, fazendo com que minerais presentes no solo sejam evaporados e entrem em contato com o oxigênio da atmosfera, transformando-se em óxidos que também ficam em suspensão no ar, podendo ser introduzidos no organismo humano durante a respiração $[\underline{7}, \underline{8}]$ e atingir até as porções mais distais das vias aéreas, onde ocorrem as trocas gasosas nos pulmões [9].

A cidade de Cuiabá, capital mato-grossense, possui topografia suave com ocorrência de extensos chapadões à sua borda, caracterizando-a como uma depressão relativa, o que ocasiona situações de estabilidade atmosférica e dificulta a dispersão de poluentes [10]. Somado a isso, no período de seca, que compreende os meses de junho a outubro, apresenta clima quente (com apenas alguns dias frios no inverno) e baixa umidade do ar [11], o que contribui para a permanência dos poluentes sobre a cidade, a qual tem sofrido com índices críticos de poluentes durante esse período, apresentando concentrações acima do estabelecido pela Resolução CONAMA n ${ }^{\circ}$ 03/1990 [12,13], o que afeta diretamente a saúde e o bem-estar da população.

Considerando a relevância do tema e as poucas informações encontradas a esse respeito para a cidade de Cuiabá e região, neste trabalho foi realizada a caracterização morfológica e química do material particulado suspenso na cidade nos meses de julho e setembro do ano de 2012, utilizando-se as técnicas de Microscopia Eletrônica de Varredura acoplada a um sistema de análise de raios X (MEV/SEDX) [14, 15$]$ e Fluorescência de Raios X por Dispersão em Energia(EDXRF) [1ㅁ, 17, $\underline{18]}$.

\section{MATERIAIS E MÉTODOS}

Para este estudo foram selecionados dois pontos dentro da área urbana da cidade para coleta de amostras: um na Estação Meteorológica Mestre Bombled, do Campus da Universidade Federal de Mato Grosso, localizada na Av. Fernando Corrêa da Costa, $n^{\circ} 2367$, no bairro Boa Esperança (1536'40'S e 56 01'33'”O) - região leste de Cuiabá; e outro na $13^{\mathrm{a}}$ Brigada de Infantaria Motorizada, localizada na Av. Historiador Rubens de

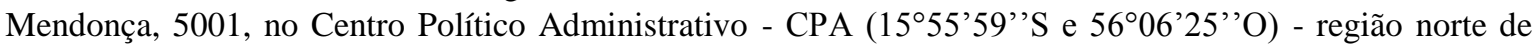
Cuiabá. Foram escolhidos estes pontos por se tratarem de locais em nível do solo (altura do indivíduo), e que não apresentavam interferentes físicos como árvores e edificações, além de serem seguros e possuírem disponibilidade de rede elétrica para a instalação do equipamento.

\subsection{Amostragem do material particulado}

As amostragens do material particulado total foram realizadas utilizando-se um amostrador de grande volume, da marca Energética, modelo AGV PTS, o qual possui bomba de sucção a vazão constante [19], utilizando filtros de fibra de vidro com eficiência de coleta de $99 \%$ para $0,3 \mu \mathrm{m}$. As amostras foram coletadas nos meses de julho e setembro de 2012, totalizando quatro amostras com período de amostragem de $24 \mathrm{~h}$ cada. 


\subsection{Caracterização química do material particulado}

A caracterização química foi realizada pela técnica de EDXRF, no Laboratório Multiusuário de Técnicas Analíticas (LAMUTA/UFMT), utilizando um equipamento da marca Shimadzu, modelo EDX-700HS, equipado com tubo de $\mathrm{Rh}$ e detector de $\mathrm{Si}(\mathrm{Li})$ resfriado por nitrogênio líquido. Foram empregados dois canais analíticos durante o processo: o primeiro para a quantificação de elementos químicos do $\mathrm{Na}$ ao Sc, com tensão no tubo de $15 \mathrm{kV}$, e o segundo para a quantificação de elementos do Ti ao U, com tensão de $50 \mathrm{kV}$. As medidas foram realizadas em ar, com tempo de aquisição de $100 \mathrm{~s}$ por canal analítico, em quintuplicata para cada amostra. Os espectros foram interpretados por meio do programa EDX-Software da Shimadzu®, utilizando o método Qual-Quant FP (método analítico por parâmetros fundamentais que se utiliza de padrões internos) e por curva de calibração para os elementos $\mathrm{Al}, \mathrm{Cu}$ e Fe. A precisão das quantificações pelo método Qual-Quant FP foi verificada por meio da análise por curvas de calibração de amostras conhecidas (padrões externos) de composição próxima à das amostras estudadas.

\subsection{Preparo dos padrões externos e curvas de calibração}

O preparo dos padrões externos foi realizado no Laboratório de Análise Físico-Química de Água e Resíduos (LAFQAR/UFMT), e foram obtidos por meio da síntese de misturas de pós nanoestruturados pelo método de gel-combustão [20, 21, 22] partindo dos seguintes reagentes: nitrato de alumínio nonahidratado, nitrato de bário, nitrato de cálcio tetrahidratado, nitrato de cobre II trihidratado, nitrato de ferro III nonahidratado e nitrato de zinco hexahidratado.

Este método foi escolhido por apresentar versatilidade, reprodutibilidade, homogeneidade e boa quantidade de amostra produzida por síntese $[\underline{20}, \underline{21}, \underline{22}, \underline{23}]$, além de produzir materiais nanoestruturados, garantindo uma maior similaridade ou equivalência entre o material particulado presente nas amostras e o utilizado para confecção dos padrões, assegurando uma maior confiabilidade na análise por EDXRF.

Para a síntese foram preparadas seis soluções de concentrações diferentes e conhecidas, cada uma contendo todos os reagentes mais o combustível (glicina), na proporção de 5 mol de combustível para cada mol de metal. Em seguida, acrescentou-se água destilada a cada solução até que se completasse um volume total de $300 \mathrm{~mL}$, sendo, então, colocadas sobre uma chapa aquecedora com cerca de $210{ }^{\circ} \mathrm{C}$ de temperatura, na qual ocorreu a redução de volume seguida da etapa de gelificação-espumificação e posterior combustão. As sínteses foram regidas segundo a reação apresentada a seguir:

a $\mathrm{Fe}\left(\mathrm{NO}_{3}\right)_{3} * 9 \mathrm{H}_{2} \mathrm{O}+b \mathrm{Ca}\left(\mathrm{NO}_{3}\right)_{2} * 4 \mathrm{H}_{2} \mathrm{O}+\mathrm{c} \mathrm{Al}\left(\mathrm{NO}_{3}\right)_{3} * 9 \mathrm{H}_{2} \mathrm{O}+d \mathrm{Cu}\left(\mathrm{NO}_{3}\right)_{2} * 3 \mathrm{H}_{2} \mathrm{O}+$ e Ba $\left(\mathrm{NO}_{3}\right)_{2}+f$ $\mathrm{Zn}\left(\mathrm{NO}_{3}\right)_{2} * 6 \mathrm{H}_{2} \mathrm{O}+\mathrm{g} \mathrm{C}_{2} \mathrm{H}_{5} \mathrm{NO}_{2} \rightarrow h \mathrm{Fe}_{2} \mathrm{O}_{3}+i \mathrm{CaO}+j \mathrm{Al}_{2} \mathrm{O}_{3}+k \mathrm{CuO}+l \mathrm{BaO}+m \mathrm{ZnO}+n \mathrm{CO}_{2}+o \mathrm{H}_{2} \mathrm{O}+$ p $\mathrm{N}_{2}$

Ao término da combustão, os materiais obtidos foram calcinados a $600^{\circ} \mathrm{C}$ durante $2 \mathrm{~h}$, para a eliminação da matéria orgânica ainda existente. Posteriormente foram homogeneizados em um almofariz de ágata.

Em seguida, foram preparadas soluções com cada um dos seis pós sintetizados, visando a confecção dos padrões pela filtração das soluções, com o intuito de obter uma distribuição homogênea do material sintetizado por toda a superfície do filtro, fator importante para a elaboração das curvas de calibração.O filtro utilizado neste procedimento foi o mesmo usado na amostragem, além de terem sido empregados valores de quantidade de massa por área similares aos obtidos nas amostragens, de maneira a assegurar uma maior similaridade entre as amostras e os padrões. Foram preparados quatro filtros (quadruplicata) para cada padrão, e então, realizadas as análises dos padrões para a elaboração das curvas de calibração, utilizando-se as mesmas condições analíticas já descritas para as amostras. As intensidades integradas dos picos e as curvas de calibração para os óxidos de $\mathrm{Al}, \mathrm{Ba}, \mathrm{Ca}, \mathrm{Cu}, \mathrm{Fe}$ e $\mathrm{Zn}$ foram montadas com o auxílio do programa Origin ${ }^{\circledR}$. Esse procedimento foi necessário para averiguar a confiabilidade das quantificações realizadas pelo método QualQuant FP para os demais óxidos, que não dispunham de padrões para a elaboração de curva de calibração.

\subsection{Caracterização morfológica das partículas}

A caracterização morfológica das partículas foi obtida pela técnica de Microscopia Eletrônica de Varredura (MEV), no Laboratório de Caracterização de Novos Materiais (LACANM/UFMT). As análises foram realizadas em um equipamento da marca Shimadzu, modelo SSX-550, acoplado a um sistema de análise de raios $\mathrm{X}$, modelo SEDX. Para estudar individualmente as partículas coletadas foram retirados dos filtros o equivalente a uma área de $1 \mathrm{~cm}^{2}$ e depositada uma camada de carbono de aproximadamente $0,25 \mathrm{~nm}$ para aumentar a condutividade da amostra. O recobrimento com carbono foi feito em um equipamento da marca Shimadzu, modelo CompactCoater CC-50, por 5 min em cada amostra. 


\section{RESULTADOS E DISCUSSÃO}

As concentrações de material particulado, em $\mu \mathrm{g} / \mathrm{m}^{3}$, obtidas no mês de julho, foram 72,99 e 141,86 , e em setembro 235,95 e 306,09, na $13^{\text {a }}$ Brigada de Infantaria Motorizada e na UFMT, respectivamente, para os dois meses. Essas variações nas concentrações estão provavelmente ligadas às condições meteorológicas do período, o qual apresentou 1005,4 mm de precipitação acumulada [24], anteriormente às amostras de julho, resultando em concentrações mais baixas que as obtidas nas amostragens de setembro, previamente às quais não houve incidência de chuva [24], propiciando um maior acúmulo de partículas na atmosfera.

De uma maneira geral, observa-se que os valores obtidos para a concentração de material particulado na UFMT são superiores aos obtidos na $13^{\mathrm{a}}$ Brigada, e um fator que pode explicar esses resultados é o fluxo de carros, que na UFMT é notadamente maior, o que contribui diretamente para esse aumento por meio da emissão veicular devido à combustão e, indiretamente, por meio da ressuspensão de partículas do solo.

Como a variação da concentração do material particulado obtido nos dois pontos de coleta, entre os meses de julho e setembro, foi praticamente idêntica $\left(162,96\right.$ e $164,23 \mu \mathrm{g} / \mathrm{m}^{3}$ na $13^{\mathrm{a}}$ Brigada de Infantaria Motorizada e na UFMT, respectivamente), pode-se presumir que houve um único fator (ou conjunto de fatores) contribuindo de maneira similar em ambos os pontos de coleta, assim sendo, não pode(m) ser este(s) fator(es) dependente(s) de características ou aspectos locais de cada ponto. Desta forma, atribuímos pelo menos uma grande parte desta variação à intensa queimada ocorrida no estado nos meses de agosto e setembro/2012 (115.165 focos de queimadas [1] , o que corresponde a quase $67 \%$ do total de focos do ano todo), acompanhada da falta de incidência de chuva no período antecedente à segunda amostragem, facilitando a permanência em suspensão do material particulado, inclusive aquele proveniente da queima de biomassa. Vale ressaltar que no mês de julho já havia registros de focos de queimada, porém, com uma incidência menos expressiva, totalizando 12.158 focos no estado nesse mês [1].

\subsection{Composição química do material particulado}

Para a determinação elementar do material particulado foram montados padrões que permitiram gerar curvas de calibração para os óxidos dos elementos químicos $\mathrm{Al}, \mathrm{Ba}, \mathrm{Ca}, \mathrm{Cu}, \mathrm{Fe}$ e $\mathrm{Zn}$, porém puderam ser utilizadas apenas as curvas de calibração dos óxidos de $\mathrm{Al}, \mathrm{Cu}$ e Fe por apresentarem precisão suficiente para utilização. Na Tabela 1 estão apresentados os parâmetros matemáticos obtidos para as curvas analíticas desses óxidos. As curvas de calibração dos óxidos de $\mathrm{Ba}, \mathrm{Ca}$ e $\mathrm{Zn}$ foram comprometidas pelo fato do filtro utilizado nas análises conter esses elementos em concentração similar ou superior ao presente nas amostras e padrões. $\mathrm{O}$ silício ( $\mathrm{Si}$ ), embora tenha sido confirmado quase na totalidade das amostras, não foi quantificado por ser o maior constituinte do filtro utilizado (de fibra de vidro), fato que comprometeu as análises quantitativas desse elemento.

Tabela 1:Parâmetros obtidos para as curvas de calibração dos óxidos de $\mathrm{Al}, \mathrm{Cu}$ e $\mathrm{Fe}$.

\begin{tabular}{c|c|c}
\hline ÓXIDOS & INTENSIDADE (contagens/ $\mu$ A) & $\mathbf{R}^{\mathbf{2}}$ \\
\hline $\mathrm{Al}_{2} \mathrm{O}_{3}$ & $10777,93_{\mathrm{x}}\left(\text { massa de } \mathrm{Al}_{2} \mathrm{O}_{3}\right)_{2}+15,16_{\times}\left(\right.$massa de $\left.\mathrm{Al}_{2} \mathrm{O}_{3}\right)+0,0004$ & 0,999 \\
\hline $\mathrm{CuO}$ & $7,39 \mathrm{x} 108_{\mathrm{x}}(\text { massa de } \mathrm{CuO})_{2}+9570,65_{\mathrm{x}}($ massa de $\mathrm{CuO})-0,020$ & 0,997 \\
\hline $\mathrm{Fe}_{2} \mathrm{O}_{3}$ & $29859,30_{\mathrm{x}}\left(\right.$ massa de $\left.\mathrm{Fe}_{2} \mathrm{O}_{3}\right)+1,244$ & 0,974 \\
\hline
\end{tabular}

Unidade de medida das massas $(\mu \mathrm{g})$.

Os resultados obtidos pela rotina Qual-Quant FP apresentaram um erro médio em relação ao valor calculado por curva de calibração de $-69,4 \%, 4,2 \%$ e $-11,5 \%$ para o $\mathrm{Al}, \mathrm{Fe}$ e $\mathrm{Cu}$, respectivamente. Um erro maior para o $\mathrm{Al}$ já era esperado pelo fato do limite de detecção para elementos com número atômico menor que 13 (Al) serem afetados por um baixo rendimento de fluorescência e outras limitações como a absorção dos raios X característicos pelo ar contido entre a amostra e o detector, pela janela de Be do detector e pela própria amostra, o que explica o erro negativo. A análise, todavia, foi suficientemente precisa para acertar a ordem de grandeza na qual o elemento está presente nas amostras. Em relação aos elementos de maior número atômico, $\mathrm{Fe}$ (26) e $\mathrm{Cu}$ (29), embora o Cu possua número atômico superior ao do $\mathrm{Fe}$, o erro apresentado para as quantificações desse elemento, em módulo, foi maior, o que deve ter ocorrido por ele estar em menor quantidade, o que propicia o aumento do erro [25]. Contudo, ainda assim o método Qual-Quant FP apresentou boa precisão em comparação às curvas de calibração, o que demonstra que para elementos de maior nú- 
mero atômico o método Qual-Quant FP é satisfatório para a determinação da concentração elementar do material particulado. Com isso, foram detectados e quantificados 10 óxidos. As concentrações obtidas pelo método Qual-Quant FP ou por curvas de calibração estão apresentadas na Tabela 2.

Nota-se que os óxidos presentes em maior quantidade nas amostras são os de $\mathrm{Al}$ e $\mathrm{Fe}$, elementos abundantes no solo da região, além do $\mathrm{Ca}$ em menor quantidade, todos associados principalmente à ressuspensão de solo, como também acontece no caso de partículas oriundas de queimadas, situação na qual é identificada a liberação de partículas de solo devido à convecção gerada pelo processo de queima [26,27]. Há também a presença, em menor proporção, de elementos típicos das emissões industriais e veiculares, como o $\mathrm{Zn}, \mathrm{S}$ e $\mathrm{Cu}$, e os provenientes da queima de biomassa, como $\mathrm{K} \mathrm{e} \mathrm{Cl}$, além do Black Carbon (BC) que não foi quantificado por limitações da técnica, mas aparece nas análises morfológicas.

Dos dados presentes na Tabela 2, nota-se que as concentrações dos óxidos de S, Zn e Sr apresentaram leve aumento em setembro em ambos os pontos de coleta, e a do Ba uma ligeira queda. Já a concentração de Fe permaneceu constante na UFMT para os dois períodos, o que não ocorreu com o $\mathrm{Al}$, fato que sugere a existência de outras fontes do mesmo, além da crostal, para esse ponto de coleta. Na $13^{a}$ Brigada, nota-se um aumento igualmente importante tanto para o óxido de $\mathrm{Fe}$ quanto para o de $\mathrm{Al}$ entre os meses de julho e setembro, o que sugere que a origem crostal é a principal fonte desses óxidos nesse ponto de coleta. A concentração de óxido de Ca praticamente dobrou na UFMT entre os meses de julho e setembro, o que pode estar ligado à maior incidência de queimadas; Entretanto, as concentrações dos óxidos de $\mathrm{K} \mathrm{e} \mathrm{Cl}$, também ligados à queima de biomassa, não apresentaram comportamento condizente com o fato observado, mostrando uma redução na concentração entre os meses citados.

Tabela 2:Concentrações elementares do material particulado coletado na cidade de Cuiabá nos meses de julho (amostras 1 e 2 ) e setembro (amostras 3 e 4 ).

\begin{tabular}{c|c|c|c|c}
\cline { 2 - 5 } ÓXIDO/ELEMENTO & $\begin{array}{c}\text { AMOSTRA 1 } \\
\text { (UFMT) }\end{array}$ & $\begin{array}{c}\text { AMOSTRA 2 } \\
\left(\mathbf{1 3}^{\mathbf{a}} \text { BRIGADA) }\right.\end{array}$ & $\begin{array}{c}\text { AMOSTRA 3 } \\
(\text { UFMT) }\end{array}$ & $\begin{array}{c}\text { AMOSTRA 4 } \\
(\mathbf{1 3} \text { - BRIGADA) }\end{array}$ \\
\hline $\mathrm{Al}_{2} \mathrm{O}_{3} *$ & 74,09 & 36,96 & 116,48 & 119,68 \\
\hline $\mathrm{SO}_{3}$ & 3,21 & 2,91 & 4,10 & 3,84 \\
\hline $\mathrm{Cl}$ & 1,05 & 0,64 & 0,70 & 0,36 \\
\hline $\mathrm{K}_{2} \mathrm{O}$ & 12,20 & 9,69 & 11,10 & 14,00 \\
\hline $\mathrm{CaO}$ & 6,80 & - & 13,10 & - \\
\hline $\mathrm{Fe}_{2} \mathrm{O}_{3} *$ & 69,69 & 22,42 & 66,62 & 81,56 \\
\hline $\mathrm{CuO} *$ & 2,02 & 0,66 & 1,34 & 1,73 \\
\hline $\mathrm{ZnO}$ & 23,30 & 18,50 & 24,60 & 23,70 \\
\hline $\mathrm{SrO}$ & 1,38 & 1,40 & 1,83 & 1,57 \\
\hline $\mathrm{BaO}$ & 10,60 & 14,20 & 8,80 & 9,30 \\
\hline
\end{tabular}

*Óxidos quantificados por curva de calibração.

Dentre os elementos identificados, dois apresentam relevância toxicológica à saúde humana, o $\mathrm{Al}$ e o $\mathrm{Cu}$, podendo causar de efeitos agudos a crônicos dependendo da concentração e tempo de exposição [28]. Vale ressaltar que, de todos os óxidos quantificados, o de $\mathrm{Al}$ apresentou a maior concentração em todas as amostras, e que o tempo de exposição da população cuiabana é alto, chegando a atingir semanas de poluição atmosférica em níveis críticos, o que agrava a situação na cidade. Além disso, há ainda a presença de BC, que possui natureza adsortiva, podendo compreender compostos carcinogênicos e diversas espécies tóxicas [29], representando desta forma grande perigo à saúde pública.

\subsection{Caracterização morfológica das partículas}

A análise dos filtros revelou uma grande variedade de partículas, que, por meio das semelhanças morfológicas e composições químicas características, foram classificadas em quatro grupos: aglomerados, placas, fi- 
brosas e arredondadas. A procura pelas partículas presentes em cada filtro foi feita de forma aleatória.

\section{Aglomerados}

Em geral, esse tipo de partícula apresentou uma morfologia de superfície rugosa com grande área específica e formas irregulares (Figura 1), aparecendo de forma majoritária nas amostras de Cuiabá, com frequência semelhante tanto nas amostras do mês de julho quanto nas de setembro. Nelas foram detectados principalmente os óxidos de Al, Si, Fe e K. Partículas com essas características normalmente são de origem crostal, como poeira, solos e partículas minerais ressuspensas do solo ou transportadas pelos ventos de regiões remotas [27, 29], como também é o caso de partículas oriundas de queimadas, fato evidenciado pela presença de K. Além desses óxidos, também foram encontrados em menor quantidade os de $\mathrm{Na}, \mathrm{Ca}, \mathrm{Zn}, \mathrm{Cu}, \mathrm{Mg}$ e $\mathrm{S}$, indicadores, na sua maioria, de emissões industriais e veiculares, e o $\mathrm{Cl}$, em geral, vinculado à queima de biomassa.

\section{Arredondadas}

As análises com EDS realizadas nas partículas arredondadas (Figura 2) indicaram a presença principalmente de C. Entretanto, algumas partículas apresentaram pequenas quantidades de óxidos de $\mathrm{Si}, \mathrm{Na}, \mathrm{Al}, \mathrm{Fe}, \mathrm{Ca}, \mathrm{S}$, e $\mathrm{K}$. Essas partículas de morfologia esferoidal e ricas em carbono são basicamente reconhecidas como fuligem ou Black Carbon [로, $\underline{30}, \underline{31}]$.

Segundo a classificação de MICIC et al. [29], elas podem ainda ser subclassificadas de acordo com a sua origem. Partículas perfeitamente esféricas (Figura 2-a) referem-se a cinzas voláteis originárias de fontes industriais, elas podem apresentar-se em formas bem definidas e amorfas ou com finas partículas de fuligem adsorvidas em sua superfície. De acordo com MICIC et al. [29], elas são identificadas como misturas de partículas carbonáceas e aluminossilicatos associados a algum outro metal, normalmente o $\mathrm{Fe}$. A presença de enxofre nessas partículas confirma sua origem industrial. Já as de superfície mais irregular (Figura 2 (b)-(c)) referem-se a partículas de BC típicas de motores de combustão interna. Nelas foram encontrados óxidos de $\mathrm{Al}, \mathrm{Na}$ e $\mathrm{Si}$, o que pode estar associado a algum processo físico que tenha gerado a aderência de partículas de solo. Partículas como a mostrada na Figura 2 (d) são típicas de fuligem gerada por queima de biomassa, esse tipo de partícula é altamente porosa, mantendo certa semelhança morfo-estrutural com seu material de origem [27]. Nessas partículas foram detectados óxidos de $\mathrm{Si}, \mathrm{Al} \mathrm{e} \mathrm{Fe}$, associados principalmente ao material de solo em suspensão, que podem estar relacionados ao movimento de convecção gerado pelo processo de queima.

As partículas esféricas foram encontradas quase que exclusivamente nas amostras de setembro, fato que provavelmente está relacionado à falta de incidência de chuva neste período, facilitando a permanência desse tipo de partícula, de menor tamanho em relação às outras, na atmosfera.

\section{Placas}

Esse tipo de partícula apresentou morfologia de superfície lisa, compacta e de aspecto translúcido (Figuras 3). Os principais óxidos encontrados nelas foram os de $\mathrm{Al}, \mathrm{Si}, \mathrm{K}, \mathrm{Fe}$ e C. No entanto, também ocorreu a presença dos óxidos de $\mathrm{Na}, \mathrm{Mg}$, S, e Ca, além de traços dos de $\mathrm{Zn}$ e Cu. Desta forma, esse grupo mostrou uma combinação de partículas tanto de origem crostal, quanto de fontes industriais e veiculares. Como essas partículas apresentaram uma maior frequência de $\mathrm{K}$ em relação aos outros grupos, é bem provável a influência de queimadas na sua formação, fator reforçado pela presença de $\mathrm{C}$ e Cl, além de apresentar ocorrência brevemente maior nas amostras de setembro. No estudo realizado por ROSASCO et al.[27], partículas semelhantes em composição e morfologia também foram relatadas e só apareceram no período em que a ocorrência de queimadas era mais evidente.

\section{Fibrosas}

Essas partículas de formato longilíneo e cilíndrico (Figura 4) apresentaram como óxidos majoritários os de $\mathrm{Al}, \mathrm{Si}, \mathrm{K}, \mathrm{Fe}$ e C , além do elemento $\mathrm{Cl}$, embora constituídas também pelos de $\mathrm{Na}, \mathrm{Mg}$ e Ca. Os óxidos presentes em maior quantidade sugerem forte influência tanto de queimadas, apresentando $\mathrm{C}, \mathrm{K} \mathrm{e} \mathrm{Cl}$ em altas concentrações, quanto de ressuspensão do solo ( $\mathrm{Si}, \mathrm{Al}$ e $\mathrm{Fe}$ ). Esse tipo morfológico ocorreu com maior incidência nas amostras de setembro e não foi relatado em outros trabalhos, o que leva a crer que é específico da região. 

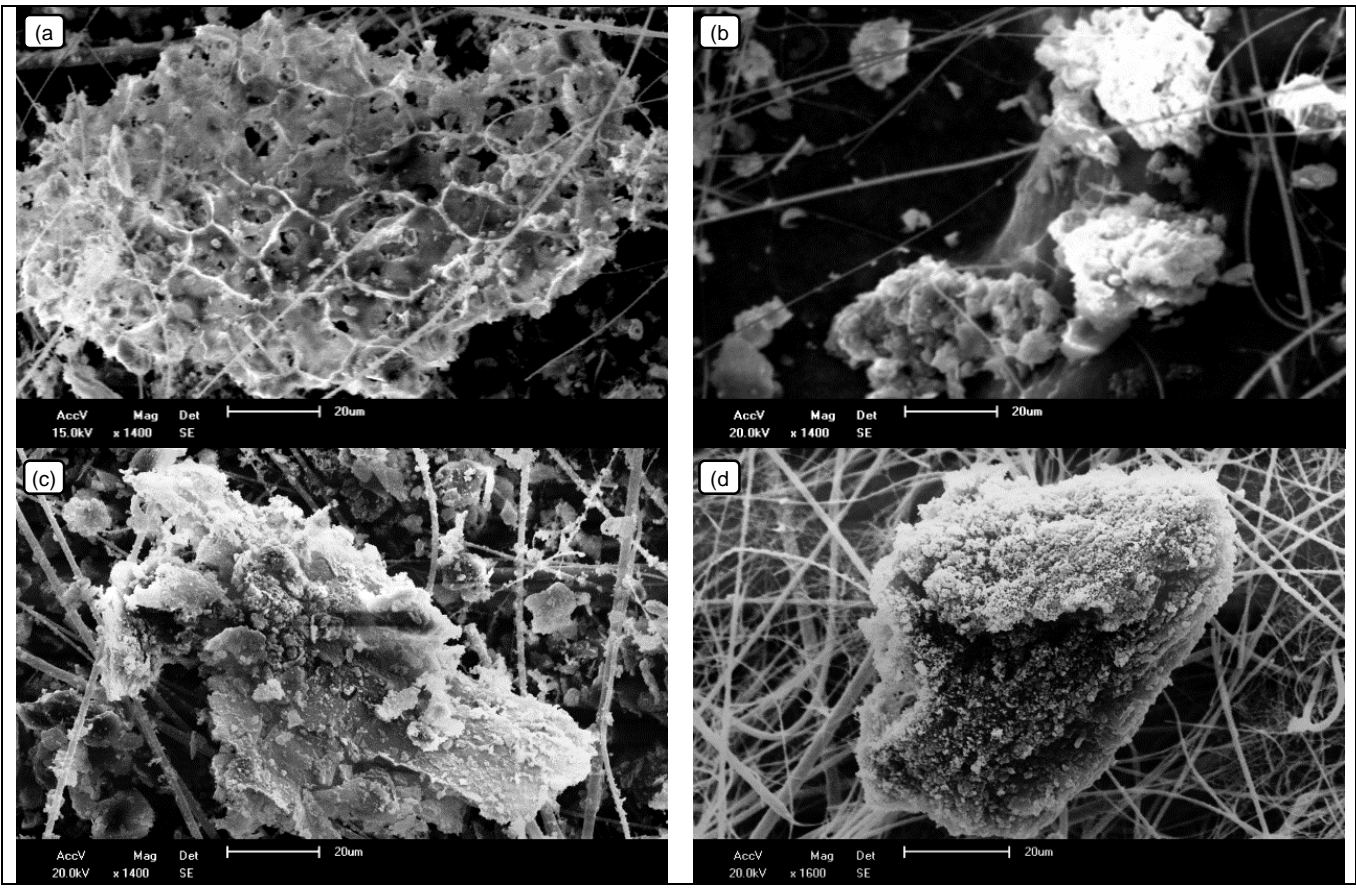

Figura 1: Imagens (SEM) de aglomerados coletados em Cuiabá: (a) e (b) partículas coletadas no mês de julho; (c) e (d) partículas coletadas no mês de setembro.
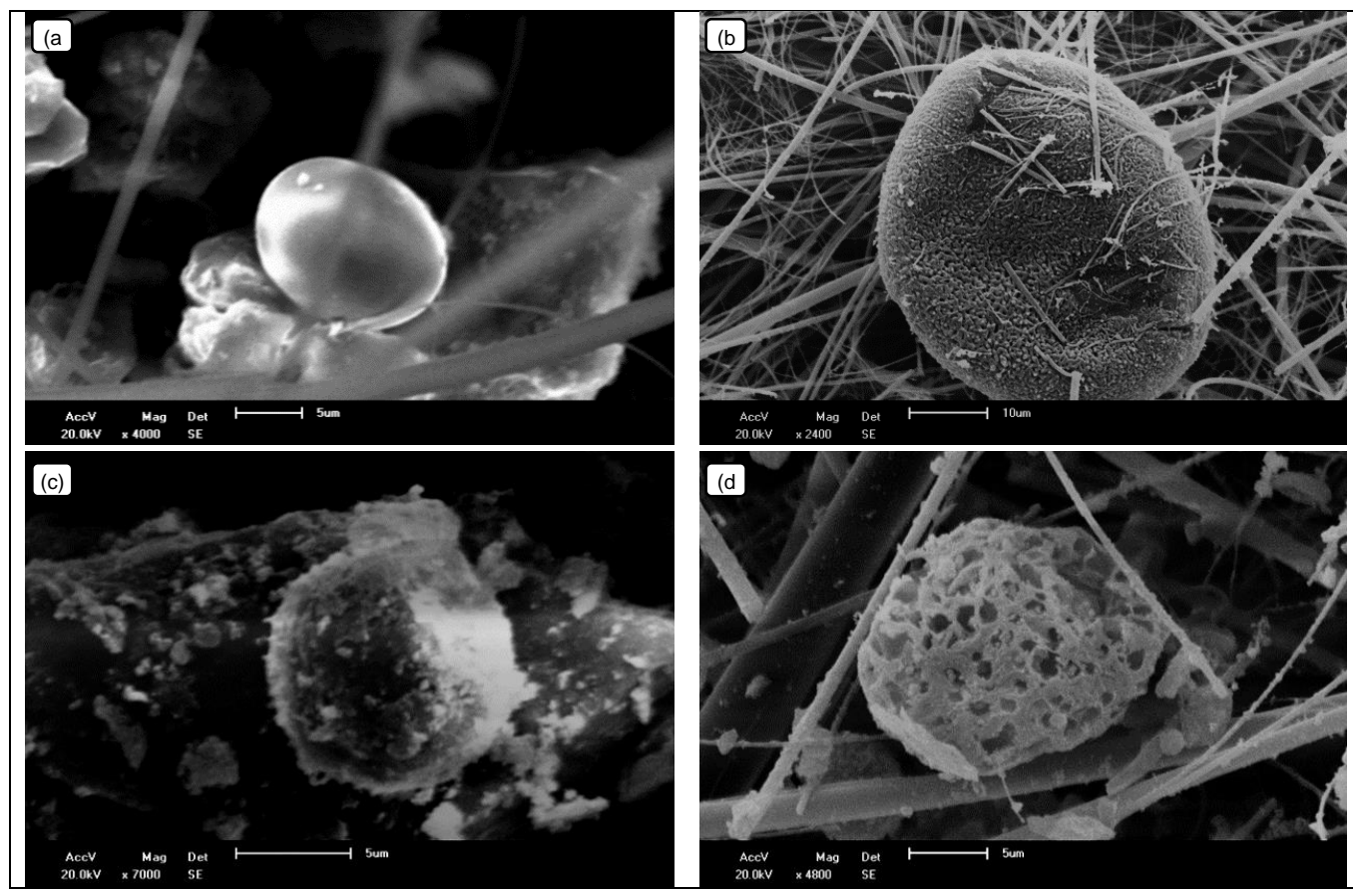

Figura 2: Imagens (SEM) de partículas arredondadas coletadas em Cuiabá: (a) partícula coletada no mês de julho, (b), (c) e (d) partículas coletadas no mês de setembro; (a) partícula típica de cinzas voláteis originárias de fontes industriais; (b) e (c) partículas típicas de motores de combustão interna; (d) partícula característica de queima de biomassa. 


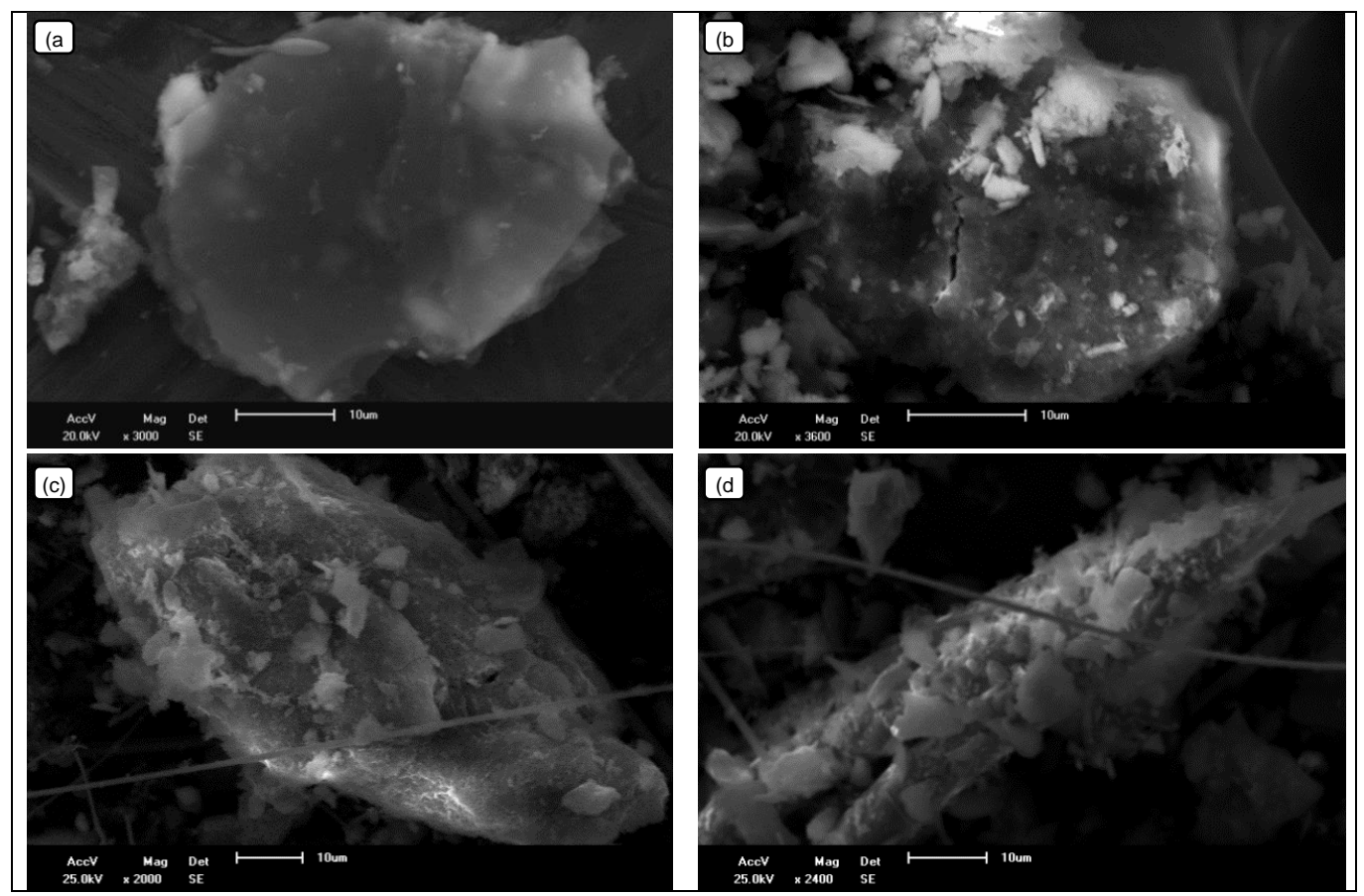

Figura 3: Imagens (SEM) de placas coletadas em Cuiabá: (a) e (b) placas coletadas no mês de julho; (c) e (d) conglomerados de placas coletados em setembro.

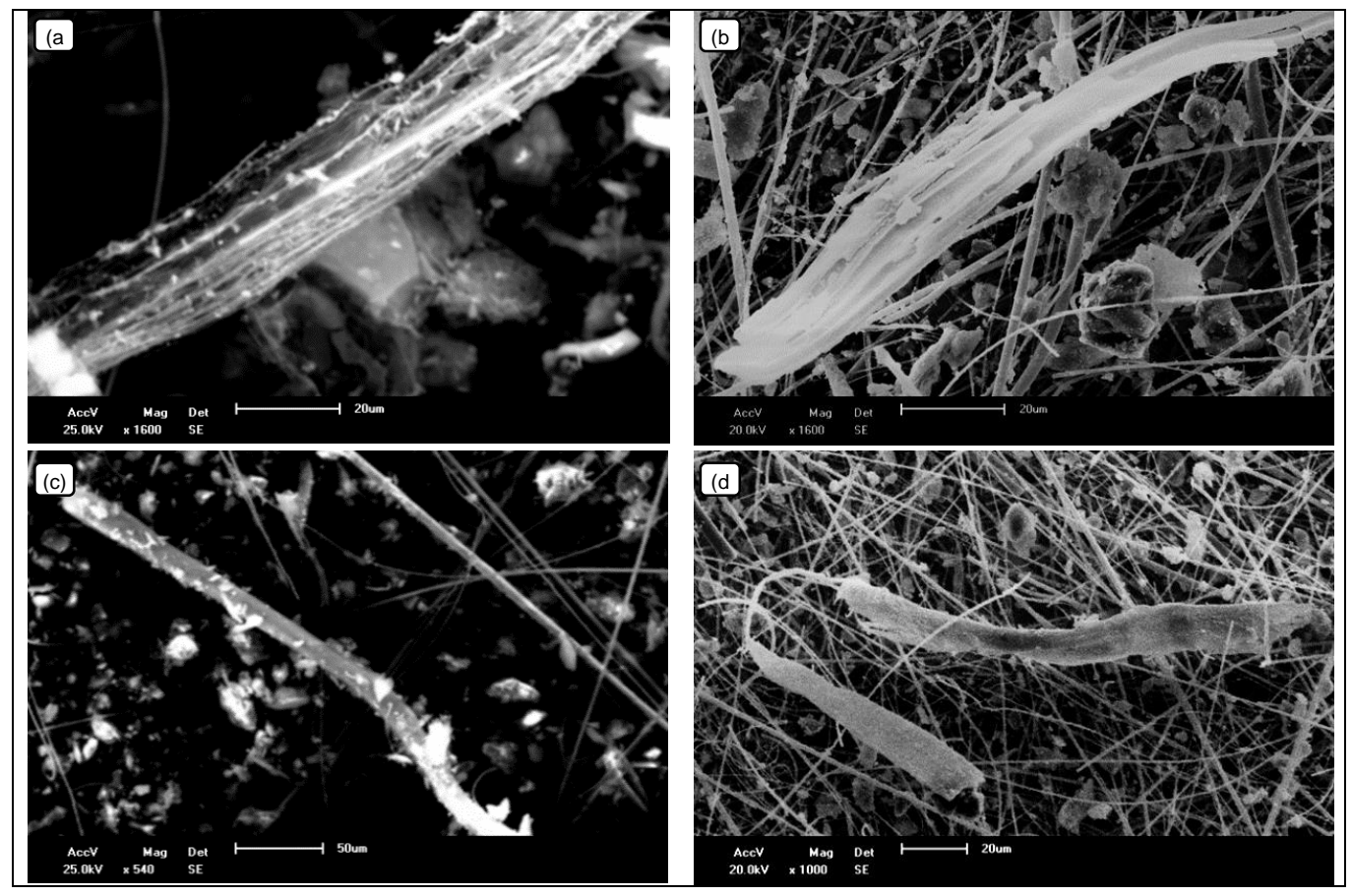

Figura 4: Imagens (SEM) de partículas fibrosas coletadas em Cuiabá: (a) partícula coletada no mês de julho; (b), (c) e (d) partículas coletadas no mês de setembro. 


\section{CONCLUSÕES}

As concentrações obtidas em Cuiabá em setembro ultrapassaram o limite estabelecido pelo CONAMA para o padrão secundário $\left(150 \mu \mathrm{g} / \mathrm{m}^{3}\right)$, sendo que a maior superou também o limite do padrão primário $\left(240 \mu \mathrm{g} / \mathrm{m}^{3}\right)$. Desta forma, fica evidente a má qualidade do ar na cidade no período de seca, resultando em efeitos adversos à vida em seu conjunto, comprometendo o conforto ambiental e bem-estar da população, bem como sua saúde, propiciando o agravamento dos casos de doenças do sistema respiratório.

Além das altas concentrações de material particulado, foram encontradas na sua composição substâncias de relevância toxicológica à saúde humana, que podem causar de efeitos agudos a crônicos dependendo da concentração e tempo de exposição, que no caso da população cuiabana é bem alto, chegando a atingir semanas de poluição atmosférica em níveis críticos no período de seca, fato que agrava ainda mais a situação na cidade.

Em relação à caracterização do material particulado encontrado na cidade, os elementos predominantes foram $\mathrm{Al}, \mathrm{Si}, \mathrm{Fe}$ e K, todos característicos de ressuspensão do solo (com exceção do K que é típico da queima de biomassa) e que podem ser emitidos por meio de queimadas e ressuspendidos por movimentações. Os outros elementos encontrados são típicos de atividades antropogênicas, tais como emissões veiculares e industriais, inclusive a agricultura e extrativismo (indústrias fortes no estado).

As análises realizadas possibilitaram identificar a morfologia e a composição química do material particulado, permitindo a quantificação de 10 elementos ( $\mathrm{Al}, \mathrm{S}, \mathrm{Cl}, \mathrm{K}, \mathrm{Ca}, \mathrm{Fe}, \mathrm{Cu}, \mathrm{Zn}, \mathrm{Sr}$ e Ba), quase todos eles na forma de óxidos, e a classificação das partículas em quatro grupos: aglomerados, arredondadas, placas e fibrosas. Fibras, como as aqui encontradas, não haviam sido relatadas anteriormente em outros trabalhos, sendo aparentemente características da região. As análises realizadas também possibilitaram a identificação das prováveis fontes de emissão desses poluentes, em geral ligados à queima de biomassa e ressuspensão do solo.

\section{AGRADECIMENTOS}

Ao Laboratório Multiusuário de Técnicas Analíticas (LAMUTA/UFMT), onde as análises de EDXRF foram realizadas; ao Laboratório de Análise Físico-química de Água e Resíduos (LAFQAR/UFMT), onde foram feitas as sínteses e preparo dos padrões; ao Laboratório de Caracterização de Novos Materiais (LACANM/UFMT), onde foram feitas as análises de MEV/EDS; e ao órgão de fomento FAPEMAT (Fundação de Amparo à Pesquisa de Mato Grosso), pelo apoio do projeto PRONEX/FAPEMAT \# 850109/2009, que possibilitou a aquisição dos reagentes utilizados na confecção dos padrões e o aprendizado sobre a técnica de síntese por gel-combustão.

\section{BIBLIOGRAFIA}

[1] INSTITUTO NACIONAL DE PESQUISAS ESPACIAIS, http://www.dpi.inpe.br/proarco/bdqueimadas/ . Acessado em fevereiro de 2013.

[2] ANDREAE, M.O., ANDERSON, B.E., BLAKE, D.R., et al., "Influence of plumes from biomass burning on atmospheric chemistry over the equatorial and tropical South Atlantic during CITE 3", Journal of Geophysical Research, v. 99, n. D6, pp. 12793-12808, Jun. 1994.

[3] YAMASOE, M.A., ARTAXO, P., MIGUEL, A.H., et al., "Chemical composition of aerosol particles from direct emissions of biomass burnings in the Amazon Basin: Water-soluble species and trace elements", Atmospheric Environment, v. 34, pp. 1641-1653, 2000.

[4] MENDONÇA, F., DANNI-OLIVEIRA, I.M., Climatologia: Noções básicas e climas do Brasil,1 ed., São Paulo, Oficina de textos, 2007.

[5] COMPANHIA AMBIENTAL DO ESTADO DE SÃO PAULO, http://www.cetesb.sp.gov.br/ar/Informa ções-Básicas/21-Poluentes. Acessado em março de 2012.

[6] ANDRADE, S.J., ANJOS, J.P., GUARIEIRO, L.L.N., et al., "Concentração de material particulado atmosférico ( $\mathrm{MP}_{10}$ e $\left.\mathrm{MP}_{2,5}\right)$ em duas regiões distintas da Bahia: uma estação de ônibus e uma ilha", In:34 Reunião Anual da Sociedade Brasileira de Química, Florianópolis/SC, AMB080, Mai. 2011.

[7] DANNI-OLIVEIRA, I.M., "Poluição do ar como causa de morbidade e mortalidade da população urbana”, R.RA'E GA, Ed.UFPR, n. 15, pp. 113-126, 2008. 
[8] GOUVEIA, N., FREITAS, C.U., MARTINS, L.C., et al., "Hospitalizações por causas respiratórias e cardiovasculares associadas à contaminação atmosférica no Município de São Paulo, Brasil”, Cadernos de Saúde Pública, v. 22, n. 12, pp. 2669-2677, Dez. 2006.

[9] BRAGA, A., PEREIRA, L.A.A.P., SALDIVA, P.H.N., "Poluição atmosférica e seus efeitos na saúde humana", Jornal Brasileiro de Pneumologia, supl. 1, pp. 10-16, 2001.

[10] MAITELLI, G.T., Uma abordagem tridimensional de clima urbano em área tropical continental: $o$ exemplo de Cuiabá-MT, Tese de D.Sc., Faculdade de Filosofia, Letras e Ciências Humanas/USP, São Paulo, SP, Brasil, 1994.

[11] MARQUES, R., A poluição atmosférica em Cuiabá: a água de chuva, deposição seca e material particulado inalável, Dissertação de M.Sc., Instituto de Ciências Humanas e Sociais/UFMT, Cuiabá, MT, Brasil, 2006.

[12] SILVA, F.C.P., Queimadas urbanas em Cuiabá - MT: origens e suas consequências socioambientais, Monografia de Graduação Tecnológica, Instituto Federal de Educação Ciência e Tecnologia de Mato Grosso, Cuiabá, MT, Brasil, 2011.

[13]SANTIAGO, A., Material particulado total suspenso na baixa atmosfera em Cuiabá-MT no período de queimadas, Dissertação de M.Sc, PPGEEA/UFMT, Cuiabá, MT, Brasil, 2013.

[14] GOLDSTEIN, J.I., NEWBURY, D.E., JOY, D.C., et.al., Scanning Electron Microscopy and X-ray Mi-

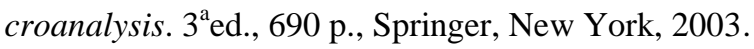

[15]SKOOG, D.A.,HOLLER, F.J.,NIEMAN, T.A., Principles of instrumental analysis, 5ed., Philadelphia, Saunders College Publishing, 1998.

[16] JENKINS, R., X-Ray Fluorescence Spectrometry, 2ª ed., Chichester, John Wiley \& Sons, 1999.

[17] GRIEKEN, R., MARKOWICZ, A., Handbook of $x$-ray spectrometry (Pratical Spectroscopy),2ed., 1016 p., CRC Press, New York, 2001.

[18] WEST, M., ELLIS, A.T., POTTS, P.J., et al., "Atomic spectrometry update - X-ray fluorescence spectrometry”. Journal of AnalyticalAtomicSpectrometry.v. 27, pp. 1603-1644, 2012.

[19] ASSOCIAÇÃO BRASILEIRA DE NORMAS TÉCNICAS (ABNT); NBR 9547, Rio de Janeiro, 1997.

[20] MAGALHÃES, G.S., Síntese e caracterização de $\mathrm{ZrO}_{2}-\mathrm{BaO}$ e $\mathrm{ZrO}_{2}-\mathrm{Y}_{2} \mathrm{O}_{3}-\mathrm{BaO}$ nanoestruturados,Dissertação de M.Sc, Instituto de Física/ UFMT, Cuiabá, MT, Brasil, 2012.

[21] LASCALEA, G.E., Obtención y Propiedades de Polvos Nanocristalinos y Materiales Cerámicos de Granos Submicrométrico Basados emCirconia, Tese de D.Sc., Instituto de Tecnología Prof. Jorge A. Sabato, Universidad Nacional de General San Martín, Argentina, 2004.

[22] FÁBREGAS, I.O., Fases Metaestables y Nuevas Propiedades em Materiales Manoestructurados Basados em $\mathrm{ZrO}_{2}$. Aplicaciones em Celdas de Combustible de Óxido Sólido, Tese de D.Sc., Facultad de Ciencias Exactas y Naturales, Universidad de Buenos Aires, Argentina, 2008.

[23] SILVA, R. C., Síntese e Caracterização de Soluções Sólidas Nanoestruturadas de Zircônia-Cálcia ( $\mathrm{ZrO} 2-\mathrm{CaO})$, Dissertação de M.Sc., Instituto de Física/UFMT, Cuiabá, MT, Brasil, 2009.

[24] INSTITUTO NACIONAL DE METEOROLOGIA, http://www.inmet.gov.br/portal/index.php?r=estaco es/estacoesAutomaticas. Acessado em setembro de 2012.

[25] SKOOG, D.A.,WEST, D.M.,HOLLER, F.J.,et al.,Fundamentos de química analítica, tradução da $8^{\mathrm{a}}$ edição norte-americana, São Paulo, Pioneira Thomson Learning, 2006.

[26] KAUFFMAN, D., CUMMINGS, D., WARD, D., "Relationships of fire, biomass and nutrient dynamics along vegetation gradient is the Brazilian Cerrado", Journal of Ecology, v. 82, n. 3, pp. 519-531, Set. 1994.

[27] ROSASCO, F.V., MARIANI, R.L., MARTINS, M.P.P., et al., "Caracterização morfológica de partículas na atmosfera de São José dos Campos - SP, utilizando microscopia eletrônica de varredura (MEV)", Geochimica Brasiliensis, v. 25,n.1, pp.25-33, 2011.

[28] AGENCY FOR TOXIC SUBSTANCES AND DISEASE REGISTRY, http://www.atsdr.cdc.gov. Acessado em setembro de 2012.

[29] MICIC, M., LEBLANC, R.M., MARKOVIC, D., et al., "Atlas of the tropospheric aerosols from Belgrade troposphere”, Fresenius Environmental Bulletin, v.12, n.9, pp.1-10, Jan. 2003. 
[30] WEINBRUCH, S., VAN AKEN, P., EBERT, M., et al., "The heterogeneous composition of working place aerosols in a nickel refinery: a transmission and scanning electron microscope study", Journal of Environmental Monitoring, v.4, n. 3, pp. 344-350, Jun. 2002.

[31] PÓSFAI, M., SIMONICS, R., LI, J., et al., "Individual aerosol particles from biomass burning in southern Africa: Compositions and size distributions of carbonaceous particles", Journal of Geophysical Research, v.108, n. D13, Jul. 2003. 